\title{
LOS CAMBIOS ORGANIZACIONALES Y LA NUBE EN EL ENTORNO TURÍSTICO: ESTUDIO DE CASO
}

\author{
Pedro R. Palos ${ }^{1}$ \\ Universidad de Extremadura \\ Facultad de Empresa, Finanzas y Turismo. \\ Departamento de Dirección de Empresas y Sociología \\ ppalos@unex.es \\ Mariano Aguayo-Camacho \\ Universidad de Sevilla \\ Facultad de CC Económicas y Empresariales. \\ Departamento de Economía Financiera y Dirección de Operaciones \\ maguayo@us.es
}

(Fecha envío: 13/05/16 - Fecha aceptación: 28/06/16)

\section{Resumen}

En los últimos años, la dependencia de las tecnologías de la información en la comercialización de destinos turísticos ha ido en aumento. La posibilidad de comercializar los destinos turísticos requiere de una infraestructura tecnológica poderosa y capaz de dar respuesta a las necesidades de cientos de miles de usuarios concurrentes. Así mismo, las necesidades de almacenamiento, procesamiento y comunicaciones se han disparado en base a estas necesidades. Una tecnología impulsora de estos nuevos servicios ha sido el cloud computing.

La adopción y uso del cloud está planteando importantes cambios organizacionales en el sector turístico y abre nuevas posibilidades a nuevos establecimientos y a establecimientos independientes que pueden competir mejor con una infraestructura TIC a costes reducidos. Este artículo presenta un estudio del caso, que ha sido planteado en cinco establecimientos hoteleros independientes de la ciudad de Sevilla. La elección se ha hecho en base al carácter independiente de los establecimientos entrevistados y la elección de la ciudad por su infraestructura en fibra óptica, que está plenamente desplegada desde hace años.

Esta investigación ha permitido conocer cuáles pueden ser las variables de influencia en el proceso de adopción y uso de la nube en el sector turístico. A su vez, este artículo detalla la importancia y la dependencia que estas aplicaciones, bajo modalidad SaaS (Software as a Service), tienen para los establecimientos y como estos pueden aumentar o disminuir sus costes en infraestructura TIC en función de la estacionalidad y ocupación.

Por último, se expone la importancia que otorgan estos establecimientos a la confianza y a la seguridad de los datos, así como que relevancia tienen para las organizaciones turísticas entrevistadas. En resumen, presentamos un artículo de naturaleza exploratoria que servirá para determinar las principales variables de influencia en futuros estudios sobre la adopción y uso de la nube en el sector turístico.

\section{Palabras clave}

cloud computing, turismo inteligente, economía digital, adopción, innovación

\section{Abstract}

In recent years, the dependency of information technology in marketing of tourist destinations has been on the rise. The possibility of marketing the tourist destinations requires a technological infrastructure powerful and able to give response to the needs of hundreds of thousands of concurrent users. Likewise, the needs of storage, processing and communications have exploded on the basis of these needs. A technology driving these new services has been cloud computing.

The adoption and use of cloud is considering significant organizational changes in the tourism sector and opens new possibilities to new establishments and independent establishments that can compete better with an ICT infrastructure to reduced costs. This article presents a case study, which has been raised in five hotels that are independent of the city of Seville. The choice has been made on the basis of the independent

\footnotetext{
${ }^{1}$ Profesor Asociado y Doctor en Economía

2 Profesor Titular y Doctor en Economía
} 
character of the establishments interviewed and the choice of the city for its infrastructure in optical fibre, which is fully deployed for years.

This investigation has allowed to know what may be the variables of influence on the process of adoption and use of the cloud in the tourism sector. In turn, this article details the importance and the dependence on these applications, under mode SaaS (Software as a Service), have for the establishments and how these can increase or decrease its costs in ICT infrastructure in function of seasonality and occupancy.

Finally, it exposes the importance they attached to these establishments to trust and data security, as well as the relevance for the tourism organizations interviewed. In summary, we present an article, of exploratory nature, which will determine the main variables of influence on future studies on the adoption and use of the cloud in the tourism sector.

\section{Key words}

cloud computing, intelligent tourism, digital economy, adoption, innovation

\section{INTRODUCCIÓN}

En sentido estricto, el cloud computing no es un concepto nuevo, se puede remontar a 1997, cuando el término fue mencionado por primera vez, pero sólo recientemente éste se ha convertido en un término de moda (Chou y Chou, 2007; Xin y Levina, 2008; Lijun, Chan y Tse, 2008). El uso del término "nube" es metafórico y por lo general apunta a un gran conjunto de recursos disponibles, hardware y software, que son de fácil acceso a través de Internet (Vaquero et al., 2009; Vouk, 2008).

Para el Instituto Nacional de Estándares y Tecnología de los Estados Unidos de América (NIST, 2011) el cloud computing es un modelo para habilitar el acceso a un conjunto de servicios computacionales (redes, servidores, almacenamiento, aplicaciones y servicios) de manera conveniente y por demanda, que pueden ser rápidamente aprovisionados y liberados con un esfuerzo administrativo y una interacción con el proveedor de servicio mínima (Forrester Research, 2009; Gartner, 2008; Buyya et al., 2009).

La tecnología cloud supone un avance, pero su adopción debe hacerse bajo un esquema modular y que ofrezca grandes facilidades de reconfiguración, en el que se puede flexiblemente acumular recursos o servicios que satisfagan las cambiantes exigencias del mercado. No obstante, el concepto aún se encuentra en España en particular y globalmente en general en su etapa más incipiente de adopción afectando de forma dispar a los dos sectores clave del tejido empresarial: la gran empresa y la Pyme.

El crecimiento que han experimentado en Internet, no sólo las aplicaciones software sino el número de usuarios que han aprovechado sus funcionalidades, ha generado que las empresas turísticas hayan invertido importantes cantidades de recursos en mantener y acompañar en ese crecimiento con infraestructuras hardware. La computación en la nube es por tanto un concepto emergente, que ha llamado mucho la atención tanto en el ámbito comercial y como en el académico.

Ese acompañamiento en el crecimiento, con recursos y medios tecnológicos ha sido constante, costoso y difícil, pero ha merecido la pena para muchas organizaciones que ven como han conseguido mejorar su competitividad y mejorar las relaciones con el cliente.

Un mayor y mejor acceso para el aprovechamiento de esos recursos, se ha convertido en estratégico para cualquier organización. Iniciativas como la virtualización, arquitecturas orientadas a servicios o el acceso multiplataforma que representan nuevos dispositivos como smartphones, tablets, etc., contribuyen decisivamente a ello.

Sin embargo, no todo son factores impulsores, también existen auténticas barreras para esta tecnología. La seguridad, la privacidad, la falta de legislación en un importante número de países o el control de la información preocupan a las organizaciones que observan estas barreras con preocupación (Frost y Sullivan, 2008).

Algunos estudios en España (ONTSI, 2014) reflejan con preocupación que se debe producir un cambio en los roles y perfiles de las personas que componen un departamento TIC. Así mismo, este mismo informe señala el desconocimiento aún existente en los sectores económicos ya que el $45,2 \%$ de las pymes españolas con web conoce el cloud computing, el $20,5 \%$ reconoce tener un sólido conocimiento de las soluciones cloud computing y su aplicabilidad en la empresa y un $24,7 \%$ ha "oído hablar" sobre la tecnología, conociendo algunos ejemplos, pero no la conoce en detalle. Por el contrario, un $54,9 \%$ de las pymes señala no conocer en absoluto la tecnología. Motivado por estas cuestiones, este estudio busca conocer las variables específicas del mercado turístico que influyen en la adopción y uso de la computación en la nube. 


\section{MARCO TEÓRICO.}

Se han identificado algunas investigaciones sobre los sistemas cloud y sus modelos de adopción. Burda y Teuteberg et al. (2014) afirman que, con frecuencia, la literatura tecnológica se centra en abordar cuestiones como la seguridad de la infraestructura cloud mediante la propuesta de nuevas arquitecturas y métodos (véase, por ejemplo, Spillner et al., 2011; Brandt et al., 2012; Wang et al., 2013). En otros casos, el eje principal de investigaciones son cuestiones relacionadas con oportunidades, costes y riesgos asociados al cloud computing (Ackermann et al, 2013; Benlian y Hess, 2011; Martens y Teuteberg, 2012), criterios de medición de la calidad del servicio (Benlian et al., 2011) o desafíos relacionados con su adopción, como la disponibilidad del servicio, su rendimiento, la falta de estándares de interoperabilidad y su dificultad de integración y personalización (Feuerlicht et al, 2011; Géczy et al., 2012). También es posible encontrar varios trabajos que inciden en la importancia de la confianza, tanto en la adopción de la tecnología cloud (Pearson, 2011; Walterbusch, Martens, y Teuteberg, 2013), como en las condiciones de privacidad en el almacenamiento de datos (lon et al., 2011).

Otras investigaciones concluyen la relevancia de aspectos tales como la incertidumbre, la compatibilidad, el apoyo de la alta dirección, la utilidad percibida, la facilidad de uso de la tecnología, la experiencia previa, las restricciones geográficas, el tamaño de la empresa, el mercado, los esfuerzos de los proveedores, la seguridad, la confianza, la influencia social y la presión de socios comerciales en la adopción de cloud computing (Alshamaila et al, 2013; Gangwar et al., 2015; Lin y Chen, 2012).

Los potenciales beneficios de la adopción de la computación en la nube pueden ser evaluados tanto en términos de ahorro financiero, como en mejoras de la gestión de los recursos informáticos. Un beneficio financiero obvio de la computación en la nube, especialmente para las empresas pequeñas y de tamaño medio, es el ahorro que representa no tener que comprar y mantener sus propias infraestructuras hardware y software (Miller, 2008).

La importante reducción de capital en la inversión en infraestructuras hardware y software, a favor de contratar servicios en la nube, ofrece a las empresas la oportunidad de adquirir las capacidades tecnológicas que puede que no hayan sido capaces de ofrecer en el pasado (Grossman, 2009).

El acceso universal a servicios software (SaaS) también puede traer beneficios financieros al no tener que pagar por el software en términos de derechos de licencia. La elasticidad de servicios en la nube también significa un manejo más flexible de los recursos, que también puede conducir a un ahorro de costes. Es decir, las empresas que pasen a prestar sus servicios a través de la nube pueden ampliar y escalar la capacidad de la demanda, pagando sólo por el uso real. PaaS ofrece un entorno de desarrollo ágil que hace que sea más fácil para los profesionales TIC desarrollar aplicaciones de forma rápida y que las adopten instantáneamente, ya que elimina la espera para el despliegue del hardware y software adecuado para las aplicaciones (Greer, 2009; Vile y Liddle, 2009).

En pocas palabras, la computación en la nube permite a las empresas, en particular las pymes y los consumidores, acceder a recursos que se podrían definir de forma coloquial como "elegidos a la carta", beneficiándose de una mayor flexibilidad y de bajos costos de la gestión de los recursos informáticos.

Pero no todo son beneficios para la empresa, ya que a pesar de la promesa de avance tecnológico que suponen los servicios en la nube, también hay obstáculos para su crecimiento y adopción. La falta constante de alta velocidad y de conexiones a Internet rápidas, es un obstáculo importante para la computación en la nube, ya que se basa en Internet para ofrecer sus servicios (Miller, 2008). La falta de estandarización de las interfaces de programas de aplicación y de plataformas tecnológicas significa que la interoperabilidad entre plataformas es pobre y las empresas no serán capaces de transferir fácilmente datos de un proveedor cloud a otro. Las empresas se enfrentan, por tanto, a proveedores de datos lock-in. Esta percepción de falta de control puede desalentar a las empresas a iniciar la adopción de la computación en la nube (Armbrust et al., 2010).

\section{METODOLOGÍA}

\subsection{El Estudio del caso}

La investigación de este artículo se basa en la adopción y uso de las tecnologías de computación en la nube o cloud computing en los hoteles independientes.

La técnica que se ha utilizado para la elaboración de esta investigación es la conocida por el estudio del caso. El método de estudio de casos, aplicado a las Ciencias Sociales, es una estrategia de investigación que se centra en la comprensión de las dinámicas que se presentan en escenarios particulares. Estos estudios contrastan diferentes fuentes y métodos de recogida de datos (ficheros, cuestionarios, entrevistas y observaciones). Una vez conseguidos los datos, estos pueden ser cuantitativos, cualitativos o ambos. El propósito de los estudios de caso es variado: brindar una descripción, verificar una teoría existente o bien generar teoría (Eisenhardt, 1989). 
Los criterios para la selección de casos son variados y resulta imprescindible ser plenamente consciente ante qué clase de caso estamos. Esto nos permitirá conocer la aportación que este estudio hará al conocimiento. Para ello debemos reflexionar sobre la situación del caso que se selecciona para su estudio en relación al universo de casos al que pertenece. En nuestro caso elegido, estamos ante un caso central, que es aquel que se da cuando un fenómeno ha sido poco o nada estudiado con anterioridad y suele ser la mejor opción centrar la atención en casos que revistan centralidad o sean muy importantes para el universo considerado. Los estudios de caso incluyen organizaciones, procesos o programas, entre otros. Se opta por el estudio de caso como estrategia de investigación cuando: a) la pregunta gira en torno al cómo y al por qué; b) el investigador tiene poco control sobre los eventos y c) el foco se encuentra en un fenómeno contemporáneo dentro de un contexto de la vida real (Forni, 2010).

En estas tres circunstancias se encontraría nuestra investigación, ya que el fenómeno de la adopción y uso del cloud es muy reciente (de 2007 en adelante), las preguntas giran a conocer cómo y porqué, ya que estamos ante una investigación que nos ayude a conocer las variables de influencia en el sector turístico y por último la tecnología cloud es absolutamente contemporánea. Se trata por tanto de una investigación empírica que investiga un fenómeno contemporáneo en el contexto de la vida real y donde se utilizan múltiples fuentes de evidencia (Yin, 1984).

El método elegido es una herramienta imprescindible en muchas áreas donde se analicen temas actuales, como es el caso de la adopción y uso de tecnología, y sobre todo brinda la oportunidad al investigador para que pueda familiarizarse con nuevos conceptos aplicándolos a un caso concreto. Por tanto, se ha desarrollado una estrategia de investigación, que se dirige hacia la comprensión, de las dinámicas presentes en contextos singulares, y combina distintos métodos para la mejora de la calidad en la recogida de muestras cualitativas y/o cuantitativas para poder verificar y generar posteriores teorías (Martínez Carazo, 2006).

Tomando como guía el estudio del caso, se llevaron a cabo entrevistas personales, con los representantes designados por los establecimientos analizados. De esta forma hemos conseguido conocer la realidad de la intención de adopción y uso del cloud computing en los hoteles de la Ciudad de Sevilla. Esta investigación de naturaleza exploratoria nos ha permitido obtener datos referentes a qué variables están relacionadas con dicha intención, el estado de adopción de esta nueva tecnología y determinar la situación de partida de futuras investigaciones.

En nuestra investigación nos marcamos como principal objetivo encontrar el modelo más adecuado de adopción de la tecnología cloud computing en el sector turístico. Para ello se realizó un análisis sobre la asimilación y aceptación de los sistemas cloud computing en 5 hoteles independientes de la Ciudad de Sevilla. Esto nos permitió comprender adecuadamente ambos procesos, así como revisar la relevancia que otorgan estos hoteles a los sistemas cloud. Para que la computación en la nube pueda crecer, es importante comprender los factores que pueden influir en su tasa de adopción por parte de los establecimientos turísticos.

\subsection{Colección de Datos}

El estudio de casos se ha extendido a 5 hoteles de la Ciudad de Sevilla. Esta muestra no resulta representativa desde un punto de vista descriptivo, pero hay que recordar qué estamos ante una investigación de naturaleza exploratoria cuyo objetivo fundamental es conocer los principales factores que influyen en la adopción del cloud en el sector turístico, en especial en los hoteles. Sin embargo, como veremos más adelante los resultados obtenidos permiten cumplir los objetivos de conocer cuáles son las variables de influencia en el uso y adopción del cloud computing en el sector turístico. Así mismo, durante las entrevistas hemos podido conocer las motivaciones que han llevado a estos establecimientos a adoptar la tecnología y de qué forma lo han llevado a cabo. Estos dos aspectos contribuyen de forma especial a la literatura sobre la materia de investigación.

El trabajo de campo se llevó a cabo entre los meses de abril y mayo de 2016. Algunas entrevistas fueron personales y otras telefónicas. Se elaboró un guion de entrevista semiestructurada, siguiendo algunos trabajos previos sobre dicha tecnología que a su vez utilizaron metodologías cualitativas de investigación en cloud computing (Yigitbasioglu, 2015). El uso de entrevistas semiestructuradas permitió a los participantes expresar libremente sus puntos de vista y a la vez permite que el entrevistador tenga la oportunidad de seguir trayectorias que puedan ser tópicas cuando sea apropiado (Crabtree, 1999).

Los datos de los hoteles fueron suministrados por la Asociación de Hoteles de la Provincia de Sevilla, que envió un email a los hoteles asociados pidiéndoles su colaboración, así como un guion de entrevista semiestructurada. El muestreo que se utilizó para recopilar los datos de este estudio fue, por tanto, a conveniencia y de recomendación o bola de nieve (Neuman, 2005). Los individuos que fueron entrevistados en representación de los hoteles fueron directivos o personas en las que éstos delegaron por razones de especialización. Los participantes tenían experiencia en cloud computing, en especial en soluciones para usuarios. Las entrevistas duraron aproximadamente 20-35 minutos y fueron transcritas durante la misma. Todos los hoteles participantes eran independientes, es decir no pertenecen a ninguna cadena u operador de viajes, contaban con menos de 100 empleados y operaban de manera internacional. 
Para conducir cada una de las entrevistas se elaboraron una serie de preguntas en tres grandes bloques, que estaban referidos a:

- Conocer las motivaciones, utilidades y servicios que usan en la nube, porqué y cómo llegaron a su adopción.

- Saber que confianza tienen en la nube y que ventajas ven la misma.

- Caracterizar el establecimiento hotelero en función de sus características económicas y tecnológicas.

La primera parte de la entrevista se centró en las razones que hicieron que estos establecimientos adoptaran la nube, así como los motivos y los beneficios que han observado desde la implantación de la computación en la nube. Esta primera parte acabó permitiéndonos conocer los servicios usados y los tipos de ficheros almacenados y más comunes, así como las razones de su uso. La segunda sección se centró en las barreras e impulsores de la tecnología respecto al sector turístico y en ella se conocieron las motivaciones que les llevan a confiar en el cloud y las ventajas asociadas, así como los motivos que les llevaron a dicha conclusión. Por último, analizamos las características definitorias del hotel desde el punto de vista económico (años de actividad, facturación y nำ de empleados).

Los entrevistados reflejaron sus percepciones basándose en sus experiencias reales con soluciones cloud computing de la modalidad SaaS (Software as a Service) o Software como Servicio, a través de las entrevistas.

\section{DISCUSIONES E IMPLICACIONES PARA EL SECTOR.}

\subsection{Experiencia en la adopción del cloud computing en el entorno turístico.}

La definición más adaptada del término ecosistema tecnológico aplicado al sector turístico sea aquella que dice que es un conjunto de agentes software inteligentes que se organizan y colaboran entre sí, para gestionar la información según las necesidades existentes en cada momento dentro del ecosistema (Chang y West, 2006). Este planteamiento sustituye a los usuarios como elementos centrales del ecosistema, de forma que este rol se le atribuye a los agentes inteligentes que, a pesar de poder llevar a cabo diferentes tareas y poseer características que les distinguen unos de otros, tienen un comportamiento predefinido y aunque puedan aprender a lo largo del tiempo y evolucionar no dejan de ser un componente software más (García-Holgado y García-Peñalvo, 2013). La industria del turismo no es ajena a los cambios tecnológicos de los últimos años, y más aún con la aparición de tecnologías como la nube, que mejoran la eficiencia en el uso de los datos y las aplicaciones. Muchas empresas y cadenas turísticas están viendo que sus ingresos dependen fuertemente de aquellas empresas que han apostado por la creación de agentes inteligentes que condicionan la elección del destino turístico o los servicios contratados por el usuario.

Así en los últimos años han sido muchas las aplicaciones que han tenido un fuerte impacto en la industria del turismo. Algunos Portales como Tripadvisor y Booking han cambiado por completo el sector y le dan una mayor participación al usuario en la elaboración de la experiencia y del producto final.

No obstante, esta aparición en el ecosistema turístico de estas aplicaciones o agentes inteligentes ha podido ocurrir en gran parte, gracias a la aparición de tecnologías como la virtualización, las arquitecturas orientadas a servicios o el acceso multiplataforma que representan nuevos dispositivos como smartphones, tablets, etc., que ha contribuido decisivamente a ello. Estas mismas tecnologías que han hecho que el cloud computing sea hoy en día una realidad, están también a disposición de los establecimientos hoteleros y permiten que estos puedan darle valor al conocimiento que generan de forma independiente. En los últimos años, el crecimiento de las capacidades tecnológicas del cloud, ha permitido almacenar y difundir la información a costes inimaginables. Hoy en día, son pocos los establecimientos turísticos que no tienen entre sus objetivos estratégicos realizar una correcta gestión del conocimiento que generan, de tal forma que este revierta en beneficios para la empresa turística.

\subsection{El cloud computing en los hoteles independientes.}

La muestra escogida hace referencia a cinco hoteles del centro de la ciudad, que han adoptado el cloud computing para tratar de mejorar algunos aspectos relacionados con la gestión, la eficiencia o la seguridad de la información y el conocimiento que generan. Todos ellos son independientes y están gestionados de forma profesional.

El primero de los hoteles es el Hotel Casa 1800 Sevilla. En pleno corazón de Sevilla, en el barrio de Santa Cruz, junto a la Catedral y a la Giralda, se encuentra el Hotel Casa 1800 Sevilla, una casa-palacio convertida en un elegante Hotel, situado en una privilegiada ubicación, junto a los más famosos monumentos, restaurantes y zonas de compras. Es un hotel de cuatro estrellas, y cuenta con 25 habitaciones decoradas de forma elegante. La distribución de las habitaciones es dispar, amén de estar situado el mismo, en un antiguo palacio del siglo XIX.

El segundo de los hoteles es el Hotel Bécquer. Edificado en la antigua casa palacio de los Marqueses de las Torres, el Hotel Bécquer propone una estancia en el centro histórico y comercial de Sevilla, con una ubicación 
a sólo 10 minutos de la Catedral. El establecimiento lleva más de 40 años abierto, y se ubica en un antiguo palacio del siglo XIX, totalmente restaurado a lo largo de la década de los años 70 . Cuentan con 134 habitaciones de diversas características, todas ellas suficientemente equipadas para su categoría. En este hotel se desarrollan numerosos actos culturales.

El tercero de los hoteles es el hotel las Casas de Judería, que se ubica en el centro de la ciudad. Es un hotel que recrea la historia, y cuenta con 134 habitaciones, todas diferentes, se entremezclan entre ellas, a través de más de sus 40 patios, sinuosos pasillos y laberínticas callejuelas interiores. Su nombre, Las Casas de la Judería, le viene, porque está enclavado en el antiguo barrio judío, a escasos metros de los principales monumentos de Sevilla: Catedral, Giralda, Reales Alcázares, Casa Pilatos y Archivo de Indias, entre otros.

El cuarto de los hoteles es el hotel Simón, un encantador hotel de 35 habitaciones localizado en la histórica calle García de Vinuesa en el centro de la ciudad. Su excelente ubicación lo sitúa a pocos minutos de la Catedral, la Plaza de Toros de la Maestranza y el Alcázar, por citar sólo algunos puntos destacados. El hotel está ubicado en un elegante edificio del siglo XVIII de estilo típico andaluz.

Y el quinto y último de los hoteles que han participado en esta investigación ha sido el Hotel Monte Triana, localizado en la zona Oeste de la ciudad, con un edificio de formas clásicas que mantiene la esencia de Andalucía. en pleno corazón del Barrio de Triana con 114 habitaciones y enfocado a turistas que necesiten visitar la Isla de la Cartuja, sede de numerosas empresas y entidades oficiales, así como el lugar donde se encuentra el Parque Temático Isla Mágica. El Hotel Monte Triana, de tres estrellas, está ubicado en el barrio de Triana, a pocos minutos a pie de la Giralda, la Catedral de Sevilla, el Real Alcázar, de la zona comercial y de Isla Mágica.

\subsubsection{El Hotel Casa 1800}

Una vez analizadas las preguntas de los diferentes bloques anteriormente referidos, estamos en condiciones de afirmar que destacan en el apartado de características, la ubicación del hotel y el trato al cliente son los dos grandes pilares donde se asienta la fortaleza de este hotel. Respecto a los aspectos más importantes a destacar, debemos indicar:

Entre las motivaciones, utilidades y servicios que usan en la nube, debemos decir que es destacable la gestión de procesos, como un buen ejemplo de cultura corporativa, donde el compromiso de la marca con sus trabajadores hace de estos los verdaderos embajadores y artífices del éxito del establecimiento. Sin embargo, el uso de las aplicaciones de la nube aún resulta escaso entre los trabajadores del hotel.

Destaca que la tecnología de la nube despierta confianza y seguridad. Como prueba de ello, este establecimiento empezó a usar la nube para almacenar ficheros y bases de datos. Tuvo conocimiento por los medios de comunicación del alcance de esta tecnología y actualmente muestra un importante grado de confianza en su uso y satisfacción. No obstante, limita el uso de la nube y no observa ventajas económicas directas o de productividad, destacando las ventajas organizacionales de manera especial y el aumento de sus capacidades informáticas sin aumentar los costes principalmente.

\subsubsection{El Hotel Bécquer}

Entre las características más destacables destaca el constante espíritu innovador del establecimiento. Este Hotel conoció las ventajas del cloud a través de las redes sociales y de cursos de formación, lo que da una idea de su capacidad para estar en línea con la innovación de procesos.

Respecto a las motivaciones, utilidades y servicios que usan en la nube, debemos decir que los ficheros, las bases de datos y la ofimática se utilizan desde la nube. Destaca que sea el único establecimiento, entre los estudiados, que despliega el cloud en los puestos de usuario. Especial importancia le otorga a las redes sociales, ya que mantiene una política clara de comunicación y reputación en la red. Usan el almacenamiento desde distintos proveedores como Google, Dropbox, Skydrive e iCloud. En cuanto al tipo de archivos que almacenan, no hacen una distinción específica de los mismos y otorgan una especial relevancia a la mejora de la productividad desde que usan la nube, a su rapidez y facilidad de uso.

En relación a la confianza que despierta esta tecnología, se refleja bastante seguridad en ella, así como en sus riesgos legales y coinciden en que han mejorado los costes desde su adopción, en especial la productividad. Por último, es de los hoteles que mayor uso de la nube hacen desde dispositivos móviles, como Smartphone o Tablets.

\subsubsection{El Hotel las Casas de la Judería}

Las motivaciones que han llevado a este establecimiento turístico a usar la nube son sobre todo las relacionadas con el almacenamiento de ficheros y bases de datos, pero también la ejecución de aplicaciones en modo SaaS: ERP (Contabilidad, Facturación) y PMS (software de gestión hotelera). 
Respecto a la confianza, otorga una gran importancia y valor a este aspecto, en especial a su seguridad, sus ventajas derivadas y al futuro que reportará. Comenzó a usar el cloud a través de una recomendación profesional y le ve muchas ventajas, destacando especialmente la disminución y ahorro de costes. Sin embargo, no usa con frecuencia la nube, si bien realiza actividades de almacenamiento de carácter periódico como copias de seguridad. Este último aspecto refleja la confianza otorgada al sistema.

Para este establecimiento el uso del cloud computing ha supuesto ventajas para la organización en cuanto a mayor flexibilidad y escalabilidad de los recursos TIC, en asegurar la continuidad de las operaciones de negocio y en una mejor competencia con empresas que tienen más recursos.

\subsubsection{El Hotel Simón}

El hotel cuenta con unas peculiares características, que hacen que lleve desarrollando desde hace años, diversas herramientas informáticas. Por ello, cuenta así mismo con una larga trayectoria en adopción de sistemas de información. La adopción de la nube se produjo a través de una oferta comercial y su principal uso se centra en una aplicación PMS (software de gestión hotelera) que funciona en modo cloud.

No utiliza la nube para almacenamiento masivo o específico de los ficheros generados en su actividad diaria, ni forma parte de su política de respaldo de datos. En cuanto a la confidencialidad de la información le otorga una importancia especial a la nube y al uso que hace de ella.

Otorga una especial confianza a la seguridad de los datos, al cumplimiento de la legislación en materia de protección de datos, a que los datos son siempre accesibles, a la rapidez de acceso y a la facilidad de uso. Respecto a los aspectos que puedan parecerle ventajas del uso de Cloud Computing destaca la disminución de costes en hardware y en la implantación de aplicaciones más rápidas.

\subsubsection{El Hotel Monte Triana}

Este establecimiento adoptó la nube a través de publicidad comercial y en concreto usa ERP (Contabilidad, Facturación) y PMS (software de gestión hotelera) bajo la modalidad SaaS. Destaca el escaso uso que hace de las soluciones de almacenamiento, si bien el número de usuarios es superior a la cuarta parte de la plantilla laboral.

La nube forma parte de la política de copias de seguridad y muestra una importante positividad hacia el futuro de esta tecnología. Así mismo, confían en aspectos como la confidencialidad, que se cumplirá la legislación en materia de protección de datos, accesibilidad, rapidez y facilidad de uso.

En cuanto a los aspectos del uso de Cloud Computing que ha supuesto ventajas para este hotel destaca la implantación de aplicaciones más rápidas, la reducción de costes, el mayor control de costes y beneficios, la mejora de la productividad, el ahorro en costes de informática, la mayor flexibilidad y escalabilidad de los recursos TIC, que asegura la continuidad de las operaciones de negocio y que la nube permite competir con empresas que tienen más recursos.

\section{CONCLUSIONES}

La intención de esta investigación ha sido conocer cuál es el estado de la adopción de la nube y que variables ejercen una mayor influencia en el sector turístico. Aunque existen diferencias entre unos autores y otros, todos están de acuerdo en un punto fundamental, hay una clara relación entre las características de un ecosistema natural y un ecosistema tecnológico en cualquiera de sus variantes y el cloud computing es un soporte fundamental para la evolución del mismo.

Comparativamente con soportes y tecnologías anteriores, se podría afirmar que a la vista de los resultados, cloud computing es una tecnología que es en sí una extensión de Internet. La tecnología de la nube actúa como elemento dinamizador de internet, ya que convierte en accesibles todos los servicios TIC. Así, los establecimientos estudiados pueden acceder a nuevos servicios, ya que el cloud globaliza los accesos a servicios TIC que antes estaban reservados sólo a aquellas empresas que antes gestionaban importantes volúmenes de reservas, y consecuentemente necesitaban disponer de importantes presupuestos con los que sólo ellas contaban. Gracias al cloud computing, los establecimientos estudiados pueden acceder a todo tipo de aplicaciones TIC necesarias para su gestión.

Sabemos, que son muchos los hoteles que ya usan a diario en sus procesos la nube y esta resulta estratégica para los mismos, pero no son siempre es así, como se puede ver en este estudio. Algunos establecimientos que fueron entrevistados llegaron a desconocer el término cloud computing y durante la entrevista reconocieron el escaso conocimiento teórico sobre esta tecnología y el modelo económico que está detrás, si bien lo utilizan a diario o para tareas estratégicas como copias de seguridad, gestionar el correo electrónico o cumplir la legislación en materia de protección de datos. 
Ha resultado llamativo la confianza otorgada en casi todos los casos, tan sólo un hotel muestra alguna reticencia a la seguridad y cumplimiento de la legislación vigente en esta materia por parte de su proveedor. Las tecnologías de la nube son una solución a medio plazo, y dependerán en gran medida de diversos factores especialmente internos, pero también externos a los establecimientos, que incidirán directamente en uno u otro sentido, como son la confianza y la relación directa con la mejora de la productividad.

La nube forma parte de la política de copias de seguridad en algunos establecimientos y en general se muestra una importante positividad hacia el futuro de esta tecnología. Así mismo, confían en aspectos como la confidencialidad, que se cumplirá la legislación en materia de protección de datos, accesibilidad, rapidez y facilidad de uso.

En cuanto a los aspectos del uso de Cloud Computing que han supuesto ventajas para los hoteles entre las respuestas obtenidas destaca la implantación de aplicaciones más rápidas, la reducción de costes, el mayor control de costes y beneficios, la mejora de la productividad, el ahorro en costes de informática, la mayor flexibilidad y escalabilidad de los recursos TIC, que asegura la continuidad de las operaciones de negocio y que la nube permite competir con empresas que tienen más recursos.

Este estudio ha contado con la importante limitación de realizarse en todo momento en hoteles de la una sola ciudad, todos ellos han sido independientes y cuentan con más de cinco años de antigüedad. Una futura línea consistirá en comparar estas entrevistas con otras realizadas en otro país o en entornos rurales con nuevos establecimientos turísticos de menos de cinco años de antigüedad.

\section{BIBLIOGRAFÍA}

Armbrust, M., Fox, A., Griffith, R., Joseph, A., Katz, R., Konwinski, A., y otros. (2010). A view of cloud computing, Communications of the ACM, Vol. 53, 50-58.

Ackermann, T., Widjaja, T., Benlian, A. y Buxmann, P. (2013). Perceived IT security risks of cloud computing: Conceptualization and scale development. 33rd International Conference on Information Systems (ICIS 2012). Orlando, FL, US.

Alshamaila, Y., Paiannidis, S. y Li, F. (2012). Cloud computing adoption by SMEs in the north east of England. A multi-perspective framework. Journal of Enterprise Information Management.

Benlian, A., Koufaris, M. y Hess, T. (2011). Service quality in software-as-a-service: Developing the SaaS-qual measure and examining its role in usage continuance. Journal of Management Information Systems, 28(3), 85-126.

Boley, H. y Chang, E. (2007). Digital ecosystems: Principles and semantics. In IEEE, International Conference on Digital Ecosystems and Technologies, Cairns, Australia, February 2007. National Research Council Canada, Ottawa, Canada.

Brandt, T., Tian, Y. y Hedwig, M. Y. (2012). Autonomic management of software as a service system with multiple quality of service classes. 20th European Conference on Information Systems (ECIS). Barcelona, Spain.

Burda, D., y Teuteberg, F. (2014). The role of trust and risk perceptions in cloud archiving. Journal of High Technology Management Research.

Buyya, R., Yeo, C.S., Venugopal, S., Broberg, J. y Brandic, I (2009). Cloud computing and emerging it platforms: Vision, hype, and reality for delivering computing as the 5th utility. Future Generation computer systems 25(6), 599-616.

Chang, E. y West, M. (2006). Digital Ecosystems A Next Generation of the Collaborative Environment. the Eight International Conference on Information Integration and Web-Based Applications \& Services 214, 3-23.

Chou, D., y Chou, A. (2007). Analysis of a new information systems outsourcing practice: software-as-a-service business model. International Journal Information Systems Change Management 2 (4), 392-405.

Crabtree, B.F., 1999. Doing Qualitative research. Sage Publications.

Eisenhardt, K. (1989). Building Theories from Case Study Research. Academy of Management Review, 14(4), 532-550.

Feuerlicht, G., Burkon, L., y Sebesta, M. (2011). Cloud computing adoption: what are the issues? System Integration, Vol. 18 No. 2, 187-192.

Forni, P. (2011). Los estudios de caso: Orígenes, cuestiones de diseño y sus aportes a la teoría social. Miríada: Investigación en Ciencias Sociales, 3(5), pp-61.

Forrester Research. (2009). Global IT Market Outlook. Obtenido en 2013 de The Global Recessions Will Slow IT Purchases Growth de A Crawl: https://www.forrester.com/Global+IT+Market+Outlook+2009/fulltext/-/ERES46676 
Frost y Sullivan. (2008). Market Demands for Hosted UC Services. Frost y Sullivan.

García-Holgado, A., y García-Peñalvo, F. J. (2013). Análisis de integración de soluciones basadas en software como servicio para la implantación de ecosistemas tecnológicos corporativos.

Gangwar, H., Date, H. y Ramaswamy, R. (2015). Understanding determinants of cloud computing adoption using an integrated TAM-TOE model. Information Technology, Journal of Entreprise Information, 28 (1), 107130.

Gartner. (2008). Assessing the Security Risks of Cloud Computing. Gartner.

Géczy, P., Izumi, N. y Kôiti, H. (2012). Cloudsourcing: managing cloud adoption. Global Journal of Business Research, Vol. 6 No. 2, 57-70.

Gretzel, U., Werthner, H., Koo, C., y Lamsfus, C. (2015). Conceptual foundations for understanding smart tourism ecosystems. Computers in Human Behavior, 50, 558-563.

Greer, M. (2009). Software as a service inflection point: Using cloud computing to achieve business agility. New York: Global Authors Publishers.

Grossman, R. (2009). The case for cloud computing. IT Professional, 11(2), 23-27. Investopedia (2016). Business ecosystem. Accedido online (24 de febrero de 2016) en $<\mathrm{http}: / /$ www.investopedia.com/terms/b/business-ecosystem.asp >.

Ion, I., Sachdeva, N., Kumaraguru, P., y Capkun, S. (2011). Home is safer than the cloud: Privacy concerns for consumer cloud storage. 7th Symposium on Usable Privacy and Security. Pittsburgh, Pennsylvania.

Lijun, M., Chan, W., y Tse, T. (2008). A Tale of Clouds: Paradigm Comparisons and Some Thoughts on Research Issues. IEEE Asia-Pacific Services Computing Conference, 468.

Lin, A., y Chen, N. (2012). Cloud computing as an innovation: Percepetion, attitude, and adoption. International Journal of Information Management Volume 32, Issue 6, 533-540.

Martens, B. y Teuteberg, F., (2012). Decision-making in cloud computing environments: A cost and risk based a roach. Information Systems Frontiers, 14(4), 871-893.

Martinez Carazo, P. (2006): "El método de estudio de caso: estrategia metodológica de la investigación científica". Pensamiento \& Gestión №: 20 pp.-pp 163-193.

Miller, M. (2008). Cloud Computing: Web-Based Applications That Change the Way You Work and collaborate online. Indiana: QUE Publishing.

Moore, J. F. (1993). Predators and prey: A new ecology of competition. Harvard Business Review, 75-86.

Neuman, W.L., (2005). Social Research Methods: Quantitative and Qualitative Approaches. Allyn and Bacon.

NIST. (2011). http://www.nist.gov/itl/cloud/ accedido el 9 de enero en 2016.

O'Connor, P. (2008): "User-Generated Content and Travel: A case Study on Tripadvisor.com". En O'Connor,P., Hopken,W., Gretzel, U. (2008): Information and communication technologies in Tourism 2008. Conference in Innsbruck, Austria.

ONTSI. (2014). El Estudio Cloud Computing. Retos y Oportunidades. Madrid. Ministerio de Industria.

Pearson, S. (2011). Toward accountability in the cloud. . IEEE Computer Society, 15(4), 64-69.

Porter, M. E., y Kramer, M. R. (2011). Creating shared value. Harvard Business Review, January-February, 6277.

Sigala, M. (2015). Applying Gamification and Assessing its Effectiveness in a Tourism Context: Behavioural and Psychological Outcomes of the TripAdvisor's Gamification Users. Asia Pacific Journal of Information Systems, 25(1), 179-210.

Spillner, J., Bombach, G., Matthischke, S., Muller, J., Tzschichholz, R. y Schill, A. (2011). Information dispersion over redundant arrays of optimal cloud storage for desktop top users. 4th IEEE International Conference on Utility and Cloud Computing (UCC). Melbourne, Australia.

Torres, J. (2011). Empresas en la Nube. Ventajas y retos del Cloud Computing. Barcelona: Libros de Cabecera.

Vaquero, L. M., Rodero-Merino, L., Caceres, J. y Lindner, M. (2009). A break in the clouds: Towards a cloud definition. Computer Communication Review, 39 (1), 50-55.

Vile, A., y Liddle, J. (2009). The Savvy guide to HPC, grid, data grid, virtualization and cloud computing. The SavvyGuideTo Ltd.

Vouk, M. (2008). Cloud computing - issues, research and implementations. Journal of Computing and Information Technology. 16, 235-246. 
Walterbusch, M., Martens, B. y Teuteberg, F. (2013). Evaluating cloud computing services from a total cost of ownership perspective. Manage. Res. Rev. 36, 613-638.

Wang, C., Chow, S., Wang, Q., Ren, K. y Lou, W., (2013). Privacy-preserving public auditing for secure cloud storage. IEEE Transactions on Computers, 62(2), 362-375.

Werthner, H. (2003). Intelligent Systems in Travel and Tourism. In: Proceedings of International Joint Conference on Artificial Intelligence (IJCAI 2003), Acapulco, Mexico, August 9-15, 2003 (pp. 1620-1625). Accedido online (24 de febrero de 2016) en <http://ijcai.org/Past\%20Proceedings/IJCAI-2003/PDF/286.pdf>.

Werthner, H., Alzua-Sorzabal, A., Cantoni, L., Dickinger, A., Gretzel, U., Jannach, D., et al. (2015). Future research issues in IT and tourism. Journal for Information Technology and Tourism. http://dx.doi.org/10.1007/s40558-014-0021-9.

Werthner, H., \& Klein, S. (1999). Information technology and tourism: A challenging relationship. Wien: SpringerVerlag.

Werthner, H., \& Ricci, F. (2004). E-commerce and tourism. Communications of the ACM, 47(12), 101-105.

Xin, M. y Levina, N. (2008). Software-as-a Service Model: Elaborating Client-Side Adoption Factors. International Conference on Information Sysytems ICIS. Paris.

Yin, R. (1984). Case Study Research. Design and Methods. Thousand Oaks, Ca.: Sage Publications.

Yigitbasioglu, O. M. (2015). The role of institutional pressures and top management support in the intention to adopt cloud computing solutions. Journal of Enterprise Information Management, 28(4), 579-594. 